CUBO A Mathematical Journal

Vol.17, No 02, (97-122). June 2015

\title{
Spacetime singularity, singular bounds and compactness for solutions of the Poisson's equation
}

\author{
Carlos Cesar Aranda \\ Blue Angel Navire research laboratory, \\ Rue Eddy 113 Gatineau, QC, Canada \\ carloscesar. aranda@gmail.com
}

\begin{abstract}
A black hole is a spacetime region in whose interior lies a structure known as a spacetime singularity whose scientific description is profoundly elusive, and which depends upon the still missing theory of quantum gravity. Using the classical weak comparison principle we are able to obtain new bounds, compactness results and concentration phenomena in the theory of Newtonian potentials of distributions with compact support which gives a suitable mathematical theory of spacetime singularity. We derive a rigorous renormalization of the Newtonian gravity law using nonlinear functional analysis and we have a solid set of astronomical observations supporting our new equation. This general setting introduces a new kind of ill posed problem with a very simple physical interpretation.
\end{abstract}

\section{RESUMEN}

Un hoyo negro es una región espacio-temporal en cuyo interior hay una estructura llamada singularidad espacio-temporal cuya descripción científica es difícil de encontrar, y que depende de la aún inexistente teoría de la gravedad cuántica. Usando el clásico principio de comparación débil, aquí probamos nuevas cotas, resultados de compacidad y fenómenos de concentración en la teoría de potenciales Newtonianos de distribuciones de soporte compacto, que dan una teoría matemática adecuada de la singularidad espacio-temporal. Derivamos una rigurosa renormalización de la ley de gravitación Newtoniana usando análisis funcional no lineal y tenemos un contundente conjunto de datos de observaciones astronómicas que apoyan nuestra nueva ecuación. Este marco general introduce una nueva forma de problema mal-puesto con una interpretación física muy simple.

Keywords and Phrases: Black hole, spacetime singularity, quantum field theory, Newtonian potentials, elliptic equations, compact imbedding, Sobolev's spaces.

2010 AMS Mathematics Subject Classification: 35J25, 35J60, 35J75. 


\section{Introduction.}

In [2, 3], the authors introduced a new concentration phenomena for the Poisson's equation using techniques from nonlinear functional analysis. In this article we are concerned with several simple consequences of this new concentration of compactness results. Using the classical theory of Newtonian potentials of distributions with compact support we are able to derive concentration of compactness for Newtonian potentials with singular behaviour. For a review of this topic see [10].

Newtonian potentials are useful in the description of gravity fields of celestial bodies [5, 20, 21. Today black holes in gravity theory and astronomy plays a central role 8 , 12, 15, 27, 30, 31, 33, [40, 46]. The interior of a black hole is usually called spacetime singularity [7, 31. In [3] we obtain the existence of a sequence $\left\{\mathfrak{P}_{j}\right\}_{j=1}^{\infty} \in \mathrm{C}^{2}(\Omega)$ for any $\Omega$ bounded domain in $\mathbb{R}^{N}$ such that $-\lim _{j \rightarrow \infty} \Delta \mathfrak{P}_{j}=\infty$ uniformly on $\Omega$ and $0<\mathfrak{P}_{j}(x) \leq \mathfrak{P}_{j+1}(\mathrm{x}) \leq$ Cte for all $x \in \Omega$. This sequence proof that it is possible to do rigorous treatment of divergence to infinite in the frame of Newtonian potentials extending rigorous quantum field theories on large scale [37, 38. Black holes are complicated real objects, and our Newtonian equation is a mathematical object but we have astronomical observations of supermassive black holes given a concrete physical significance to this new theoretical frame [27. This is a remarkable fact in gravity theory [13, 41].

Lemma 1.1 (Lemma 1 page 277 [11). Let $\Omega$ be an open set of $\mathbb{R}^{\mathrm{N}}, \mathrm{f} \in \mathfrak{D}^{\prime}(\Omega)$ and $\mathrm{u}$ a solution (in the sense of distribution) of Poisson's equation $\Delta \mathrm{u}=\mathrm{f}$ on $\Omega$. Then for every bounded open set $\Omega_{1}$ with $\bar{\Omega}_{1} \subset \Omega$ there exists $\mathrm{f}_{1} \in \mathscr{E}^{\prime}$ the space of distributions on $\mathbb{R}^{\mathrm{N}}$ with compact support, such that

$$
f_{1}=f \text { on } \Omega, u=\text { the Newtonian potential of } \mathrm{f}_{1} \text { on } \Omega_{1} \text {. }
$$

Therefore for $\Omega_{1} \subset \Omega$ and our sequence $\left\{\mathfrak{P}_{j}\right\}_{j=1}^{\infty}$ there exists a sequence $\left\{\mathrm{f}_{1, j}\right\}_{j=1}^{\infty} \in \mathscr{E}^{\prime}$ the space of distributions on $\mathbb{R}^{N}$ with compact support, such that

$$
f_{1, j}=\Delta \mathfrak{P}_{j} \text { on } \Omega, \mathfrak{P}_{j}=\text { the Newtonian potential of } f_{1, j} \text { on } \Omega_{1} \text {. }
$$

We have associated to each pair $\left\{\mathfrak{P}_{j}, \Omega_{1}\right\}$ a gravitational Newtonian potential defined on all $\mathbb{R}^{\mathrm{N}}$. Using this lemma we have a very simple Newtonian interpretation of the interior of a black hole. We have a set of solid astronomic observations supporting our new equation. 


\begin{tabular}{|c|c|c|}
\hline Black hole name & Solar mass $(\operatorname{sun}=1)$ & References \\
\hline Holmberg 15A & 170.000 .000 .000 & 23 \\
\hline S5 $0014+813$ & 40.000 .000 .000 & 16 \\
\hline SDSS J085543.40-001517.7 & 25.000.000.000 & 47 \\
\hline APM $08279+5255$ & 23.000 .000 .000 & 35 \\
\hline NGC 4889 & 21.000.000.000 & 25 \\
\hline Central black hole of Phoenix cluster & 20.000 .000 .000 & 26 \\
\hline SDSS J07451.78+734336.1 & 19.500 .000 .000 & 47 \\
\hline OJ 285 primary & 18.000 .000 .000 & 39 \\
\hline SDSS J08019.69+373047.3 & 15.140 .000 .000 & 4 \\
\hline SDSS J115954.33+20192.1 & 14.120 .000 .000 & 47 \\
\hline SDSS J075303.34+423130.8 & 13.500 .000 .000 & 47 \\
\hline SDSS J081855.77+095848.0 & 12.000 .000 .000 & 47 \\
\hline SDSS J0100+2802 & 12.000 .000 .000 & 44 \\
\hline SDSS J082535.19+512706.3 & 11.220 .000 .000 & 47 \\
\hline SDSS J013127.34-0321000.1 & 11.000 .000 .000 & $\diamond$ \\
\hline Central black hole of RX J1532.9+3021 & 10.000.000.000 & $\diamond$ \\
\hline QSO B2126-158 & 10.000 .000 .000 & 16 \\
\hline SDSS J015741.57-010629.6 & 9.800 .000 .00 & 47 \\
\hline NGC 3842 & 9.700 .000 .000 & 25 \\
\hline SDSS J2330301.45-093930.7 & 9.120 .000 .000 & 47 \\
\hline SDSS J075819.70+202300.9 & 7.800 .000 .000 & 47 \\
\hline SDSS J080956.02+50200.9 & 6.450 .000 .000 & 47 \\
\hline SDSS J0142114.75+0023224.2 & 6.310 .000 .000 & 47 \\
\hline Messier 87 & 6.300 .000 .000 & $\diamond$ \\
\hline QSO B0746+254 & 5.000 .000 .000 & 16 \\
\hline QSO B2149-306 & 5.000 .000 .000 & 16 \\
\hline NGC 1277 & 5.000 .000 .000 & $\diamond$ \\
\hline SDSS J090033.50+421547.0 & 4.700 .000 .000 & 47 \\
\hline Messier 60 & 4.500 .000 .000 & $\diamond$ \\
\hline SDSS J011521.20+152453.3 & 4.100 .000 .000 & 47 \\
\hline QSO B0222+185 & 4.000 .000 .000 & [16] \\
\hline Hercules A (3C 348) & 4.000 .000 .000 & $\nabla$ \\
\hline SDSS J213023.61+122252.0 & 3.500 .000 .000 & 47 \\
\hline
\end{tabular}




\begin{tabular}{|c|c|c|}
\hline $\mathrm{J} 173352.23+540030.4$ & 3.400 .000 .000 & 47 \\
\hline SDSS J025021.76-075749.9 & 3.100 .000 .000 & 47 \\
\hline SDSS J030341.04-002321.9 & 3.000 .000 .000 & 47 \\
\hline QSO B0836+710 & 3.000 .000 .000 & 16 \\
\hline SDSS J224956.08+000218.0 & 2.630 .000 .000 & 47 \\
\hline SDSS J030449.85-000813.4 & 2.400 .000 .000 & 47. \\
\hline SDSS J234625.66-001600.4 & 2.240 .000 .000 & 47 \\
\hline ULAS J1120+0641 & 2.000 .000 .000 & $\diamond$ \\
\hline QSO 0537-286 & 2.000 .000 .000 & {$[16]$} \\
\hline NGC 3115 & 2.000 .000 .000 & $\diamond$ \\
\hline $\mathrm{Q} 0906+6930$ & 2.000 .000 .000 & $\diamond$ \\
\hline QSO B0805+614 & 1.500 .000 .000 & {$[16]$} \\
\hline Messier 84 & 1.500 .000 .000 & $\diamond$ \\
\hline QSO B225155+2217 & 1.000 .000 .000 & {$[16$} \\
\hline QSO B1210+330 & 1.000 .000 .000 & {$[16$} \\
\hline NGC 6166 & 1.000 .000 .000 & $\diamond$ \\
\hline Cygnus A & 1.000 .000 .000 & $\diamond$ \\
\hline Sombrero Galaxy & 1.000 .000 .000 & $\diamond$ \\
\hline Markarian 501 & 900.000.000-3.400.000.000 & $\diamond$ \\
\hline PG $1426+015$ & 467.740 .000 & 28 \\
\hline $3 \mathrm{C} 273$ & 550.000 .000 & 28 \\
\hline Messier 49 & 560.000 .000 & $\diamond$ \\
\hline PG $0804+761$ & 190.550 .000 & 28 \\
\hline PG $1617+175$ & 275.420 .000 & 28 \\
\hline $\mathrm{PG} 1700+518$ & 60.260 .000 & 28 \\
\hline NGC 4261 & 400.000 .000 & $\diamond$ \\
\hline NGC 1275 & 340.000 .000 & $\diamond$ \\
\hline 3C 390.3 & 338.840 .000 & 28 \\
\hline II Zwicky 136 & 144.540 .000 & 28 \\
\hline PG $0052+251$ & 218.780 .000 & 28 \\
\hline Messier 59 & 270.000 .000 & $\diamond$ \\
\hline PG $1411+442$ & 79.430 .000 & 28 \\
\hline Markarian 876 & 240.000 .000 & 28 \\
\hline Andromeda Galaxy & 230.000 .000 & $\diamond$ \\
\hline PG $0953+414$ & 182.000 .000 & 28 \\
\hline PG $0026+129$ & 53.700 .000 & 28 \\
\hline Fairall 9 & 79.430 .000 & 28 \\
\hline
\end{tabular}




\begin{tabular}{|c|c|c|}
\hline Markarian 1095 & 182.000 .000 & 28 \\
\hline Messier 105 & 200.000 .000 & $\diamond$ \\
\hline Markarian 509 & 57.550 .000 & 28 \\
\hline OJ 287 secondary & 100.000 .000 & 39 \\
\hline RX J124236.9-1111935 & 100.000 .000 & $\diamond$ \\
\hline Messier 85 & 100.000 .000 & $\diamond$ \\
\hline NGC 5548 & 123.000 .000 & $\diamond$ \\
\hline PG $1221+143$ & 40.740 .000 & 28 \\
\hline Messier 88 & 80.000 .000 & $\diamond$ \\
\hline Messier 81 & 70.000 .000 & $\diamond$ \\
\hline Markarian 771 & 75.860 .000 & 28 \\
\hline Messier 58 & 70.000 .000 & $\diamond$ \\
\hline PG $0844+349$ & 21.380 .000 & 28 \\
\hline Centaurus A & 55.000 .000 & $\diamond$ \\
\hline Markarian 79 & 52.500 .000 & 28 \\
\hline Messier 96 & 48.000 .000 & $\diamond$ \\
\hline Markarian 817 & 43.650 .000 & 28 \\
\hline NGC 3227 & 38.900 .000 & 28 \\
\hline NGC 4151 primary & 40.000 .000 & $\diamond$ \\
\hline $3 \mathrm{C} 120$ & 22.900 .000 & $\diamond$ \\
\hline Markarian 279 & 41.700 .000 & $\diamond$ \\
\hline NGC 3516 & 23.000 .000 & $\diamond$ \\
\hline NGC 863 & 17.700 .00 & $\diamond$ \\
\hline Messier 82 & 30.000 .000 & $\diamond$ \\
\hline Messier 108 & 24.000 .000 & $\diamond$ \\
\hline NGC 3783 & 9.300 .000 & $\diamond$ \\
\hline Markarian 110 & 5.620 .000 & $\diamond$ \\
\hline Markarian 335 & 6.310 .000 & $\diamond$ \\
\hline NGC 4151 secondary & 10.000 .000 & $\diamond$ \\
\hline NGC 7469 & 6.460 .000 & $\diamond$ \\
\hline IC $4329 \mathrm{~A}$ & 5.010 .000 & $\diamond$ \\
\hline NGC 4593 & 8.130 .000 & $\diamond$ \\
\hline Messier 61 & 5.000 .000 & $\diamond$ \\
\hline Messier 32 & $1.500 .000-5.000 .000$ & $\diamond$ \\
\hline Sagittarius A* & 4.100 .000 & $\diamond$ \\
\hline NGC 4051 & 1.300 .000 & $\diamond$ \\
\hline
\end{tabular}


references are provided.

Our function $\mathfrak{P}=\lim _{\mathfrak{j} \rightarrow \infty} \mathfrak{P}_{\mathfrak{j}}$ is very 'rough' from the point of view of regularity on Sobolev's spaces in opposition of the Poisson's equation $\Delta \mathrm{u}=0$ on $\Omega$.

Theorem 1.2 (Weyl page 118[10]). Let $\Omega \subset \mathbb{R}^{N}$ be open and $u \in \mathrm{L}_{\text {loc }}^{1}(\Omega)$ satisfy $\int_{\Omega} u \Delta v=0$ for all $v \in \mathrm{C}_{0}^{\infty}(\Omega)$. Then $\mathrm{u} \in \mathrm{C}^{\infty}(\Omega)$ and $\Delta \mathrm{u}=0$.

A simpler use of Green's identity allow us to imply the discontinuity at infinitum of the functional $\mathfrak{G}: \mathrm{C}^{2}(\bar{\Omega}) \times \mathrm{C}^{2}(\bar{\Omega}) \rightarrow \mathbb{R}$

$$
\mathfrak{G}(u, v)=\int_{\Omega}(v \Delta u-u \Delta v) d x-\int_{\partial \Omega}\left(v \frac{\partial u}{\partial n}-u \frac{\partial v}{\partial n}\right) d \gamma,
$$

where $\Omega$ is a bounded domain with $C^{1}$ boundary $\partial \Omega$. The functional $\mathfrak{G}$ for a fixed pair $(u, v) \in$ $\mathrm{C}^{2}(\bar{\Omega}) \times \mathrm{C}^{2}(\bar{\Omega})$ in a smooth bounded domain $\Omega \subset \mathbb{R}^{N}$ satisfies the Green's identity $\mathfrak{G}(\mathfrak{u}, v)=0$ (for a proof of Green's formula see page 20 [29]). This equality is a consequence of the divergence Theorem, (for a proof of the divergence Theorem stated by E. Heinz see page 46 [36], also page 570 [11]). Let us to remember several classical results:

Theorem 1.3. [Theorem 6.6 [18]] Let $\Omega$ be a $\mathrm{C}^{2, \alpha}$ domain in $\mathbb{R}^{\mathrm{N}}$ and let $\mathrm{u} \in \mathrm{C}^{2, \alpha}(\bar{\Omega})$ be a solution of the equation

$$
L u=\sum_{i, j=1}^{N} a_{i j} u_{x_{i}, x_{j}}+\sum_{i=1}^{N} b_{i} u_{x_{i}}+c u=f,
$$

where $\mathrm{f} \in \mathrm{C}^{\alpha}(\bar{\Omega})$ and the coefficients of $\mathrm{L}$ satisfy, for positive constants $\lambda, \Lambda$

$$
\begin{array}{r}
\sum_{i, j=1}^{N} a_{i j} \xi_{i} \xi_{j} \geq \lambda|\xi|^{2}, \text { for all } x \in \Omega, \xi \in \mathbb{R}^{N}, \\
\left|a_{i, j}\right|_{0, \alpha ; \Omega},\left|b_{i}\right|_{0, \alpha ; \Omega},\left|c_{i}\right|_{0, \alpha ; \Omega} \leq \Lambda .
\end{array}
$$

Let $\varphi \in \mathrm{C}^{2, \alpha}(\bar{\Omega})$ and suppose $\mathrm{u}=\varphi$ on $\partial \Omega$. Then

$$
|u|_{2, \alpha ; \Omega} \leq \mathrm{C}\left\{|\mathrm{u}|_{0, \Omega}+|\varphi|_{2, \alpha ; \Omega}+|f|_{0, \alpha ; \Omega}\right\},
$$

where $\mathrm{C}=\mathrm{C}(\mathrm{N}, \alpha, \lambda, \Lambda, \Omega)$.

$$
\begin{array}{r}
L u=D_{i}\left(a^{i j}(x) D_{j} u+b^{i}(x) u\right)+c^{i}(x) D_{i} u+d(x) u, \\
L^{*} u=D_{i}\left(a^{i j} D_{j} u-c^{i} u\right)-b^{i} D_{i} u+d u, \\
a^{i j}(x) \xi_{i} \xi_{j} \geq \lambda|\xi|^{2}, \text { for all } x \in \Omega, \xi \in \mathbb{R}^{N}, \\
\sum\left|a^{i j}(x)\right|^{2} \leq \Lambda^{2}, \\
\lambda^{-2} \sum\left(\left|b^{i}(x)\right|^{2}+\left|c^{i}(x)\right|^{2}\right)+\lambda^{-1}|d(x)| \leq \mathfrak{v}^{2},
\end{array}
$$


Theorem 1.4. [Theorem 9.11[18]] Let $\Omega$ be an open set in $\mathbb{R}^{\mathrm{N}}$ and $\mathrm{W}^{2, p}(\Omega) \cap \mathrm{L}^{\mathrm{p}}(\Omega), 1<\mathrm{p}<\infty$, a strong solution of the equation $\mathrm{Lu}=\mathrm{f}$ in $\Omega$ where the coefficients of $\mathrm{L}$ satisfy for positive constants $\lambda, \wedge$

$$
\begin{array}{r}
a^{i j} \in C^{0}(\Omega), b^{i}, c \in L^{\infty}(\Omega), f \in L^{p}(\Omega) ; \\
a^{i j} \xi_{i} \xi_{j} \geq \lambda|\xi|^{2} \text { for all } \xi \in \mathbb{R}^{N}, \\
\left|a^{i, j}\right|,\left|b^{i}\right|,|c| \leq \Lambda,
\end{array}
$$

where $i, j=1, \ldots, N$. Then for any domain $\Omega^{\prime} \subset \subset \Omega$,

$$
\|u\|_{2, p ; \Omega^{\prime}} \leq \mathrm{C}\left(\|\mathrm{u}\|_{p ; \Omega}+\|f\|_{p ; \Omega}\right),
$$

where $\mathrm{C}$ depends on $\mathrm{N}, \mathrm{p}, \lambda, \Lambda, \Omega^{\prime}, \Omega$ and the moduli of continuity of the coefficients $\mathrm{a}^{\mathrm{ij}}$ on $\Omega^{\prime}$.

Now the existence of our concentrating sequence of non negative, bounded functions $\left\{\mathfrak{P}_{j}\right\}_{j=1}^{\infty}$ is a compactness property not established and noncontradictory with the statements in Theorems 1.3 and 1.4. This is a singular property that using very elementary techniques, allow us to obtain new bounds and several interesting results related to the Newtonian potential in the theory of distributions [11. Moreover we built a sequence $\mathfrak{F}_{j}:[a, b] \rightarrow[0, \infty)$ satisfying $\lim _{j \rightarrow \infty} \mathfrak{F}_{j}^{\prime \prime}(x)=$ $-\infty$ in measure, Cte $\geq \mathfrak{F}_{j+1} \geq \mathfrak{F}_{j} \geq 0$ and each $\mathfrak{F}_{j}$ non increasing on $[\mathrm{a}, \mathrm{b}]$. By considering $\mathfrak{F}_{\mathfrak{j}}\left(x_{1}, x_{2}, \ldots, x_{N}\right)=\mathfrak{F}_{\mathfrak{j}}\left(x_{1}\right)$ we obtain a sequence for bounded smooth domains in $\mathbb{R}^{N}, N \geq 2$ with similar properties.

\section{Preliminaries.}

The results of this section are contained in $[3]$. For the sake of the readability, we stated and prove it here again.

Lemma 2.1. Let $\mathrm{B}(0, \mathrm{R})$ be a ball of radius $\mathrm{R}>0$ in $\mathbb{R}^{\mathrm{N}}, \mathrm{N}>2$. Consider the singular nonlinear elliptic equation

$$
\begin{aligned}
-\Delta \mathfrak{P}_{j} & =\mathfrak{H}_{j}\left(\mathfrak{P}_{j}\right) & \text { in } \mathrm{B}(0, \mathrm{R}) \\
\mathfrak{P}_{j} & =0 & \text { on } \partial \mathrm{B}(0, \mathrm{R}) .
\end{aligned}
$$

where $\mathfrak{H}_{j}:(0, \infty) \rightarrow(0, \infty)$ is locally Hölder continuous function

$$
\mathfrak{H}_{\mathfrak{j}}(\mathrm{s})= \begin{cases}\mathrm{s}^{-j} & \text { if } 0<\mathrm{s}<1 \\ \mathrm{~s}^{-1} & \text { if } \mathrm{s} \geq 1\end{cases}
$$

Then the next properties holds:

(i) The sequence $\left\{\mathfrak{P}_{j}\right\}_{j=1}^{\infty} \in \mathrm{C}^{2}(\mathrm{~B}(0, \mathrm{R})) \cap \mathrm{C}(\overline{\mathrm{B}(0, \mathrm{R})})$ are radial functions with $\frac{\partial \mathfrak{P}}{\partial \mathrm{r}}<0$.

(ii) The sequence $\left\{\mathfrak{P}_{j}\right\}_{j=1}^{\infty}$ satisfies $\mathfrak{P}_{\mathfrak{j}} \leq \mathfrak{P}_{\mathfrak{j}+1}$.

(iii) The sequence $\left\{\mathfrak{H}_{\mathfrak{j}}\left(\mathfrak{P}_{\mathfrak{j}}\right)\right\}_{\mathfrak{j}=1}^{\infty}$ satisfies $\mathfrak{H}_{j}\left(\mathfrak{P}_{\mathfrak{j}}\right) \leq \mathfrak{H}_{j+1}\left(\mathfrak{P}_{\mathfrak{j}+1}\right)$.

(iv) The sequence $\left\{\mathfrak{P}_{j}\right\}_{j=1}^{\infty}$ satisfies $w \leq \mathfrak{P}_{j} \leq e$, where $-\Delta v=v^{-1}$ in $\mathrm{B}(0, \mathrm{R}), v=0$ on $\partial \mathrm{B}(0, \mathrm{R})$, 
$-\Delta e=e^{-1}$ in $\mathrm{B}(0, \mathrm{R}), e=1$ on $\partial \mathrm{B}(0, \mathrm{R})$ and $-\Delta w=e^{-1}$ in $\mathrm{B}(0, \mathrm{R}), w=0$ on $\partial \mathrm{B}(0, \mathrm{R})$.

Proof. The enunciate (i) is a consequence of classical results on radial symmetry. The points (ii) and (iii) have been stated at [2, 3] and the point (iv) is proved in [1].

Theorem $2.2(\underline{3})$. Let $\mathrm{B}(0, \mathrm{R}) \subset \mathbb{R}^{\mathrm{N}}$, a ball of radius $\mathrm{R}$, with $\mathrm{N} \geq 3$. Then there exists a sequence of radial, nonnegative and bounded functions $\left\{\mathfrak{P}_{j}\right\}_{j=1}^{\infty}$ and $0 \leq \mathrm{R}_{\mathcal{O}}<\mathrm{R}$ such that

$$
-\lim _{j \rightarrow \infty} \Delta \mathfrak{P}_{j}=\infty \text { on } \mathrm{A}\left(\mathrm{R}_{0}, \mathrm{R}\right) \text {, }
$$

where $A\left(R_{0}, R\right)$ is the annulus of external radius $R$ and internal radius $R_{0}$. Moreover $\mathfrak{P}_{j} \in$ $\mathrm{C}^{\infty}\left(\mathrm{A}\left(\mathrm{R}_{0}, \mathrm{R}\right)\right)$ and $\mathfrak{P}_{j} \leq \mathfrak{P}_{j+1}$.

Proof. Our proof is a reductio ad absurdum procedure. Let us to consider

$$
\mathfrak{P}=\lim _{j \rightarrow \infty} \mathfrak{P}_{j}
$$

Now if there exists no sequence such that it is stated in our Theorem 2.2, then

$$
\lim _{r \nearrow \mathbb{R}} \mathfrak{P}(r) \geq 1
$$

because if $\lim _{\mathrm{r}}>\mathrm{R} \mathfrak{P}(\mathrm{r})<1$ for a all nonnegative and small enough $\epsilon$, there exist $\delta>0$ such that $\mathfrak{P}(r) \leq 1-\epsilon$ for all $r \in(R-\delta, R)$. Therefore $\mathfrak{P}_{j}(r) \leq \mathfrak{P}(r) \leq 1-\epsilon$ for all $r \in(R-\delta, R)$ and

$$
\begin{aligned}
-\lim _{j \rightarrow \infty} \Delta \mathfrak{P}_{j} & =\lim _{j \rightarrow \infty} \mathfrak{H}_{j}\left(\mathfrak{P}_{j}\right) \\
& =\lim _{j \rightarrow\left(\mathfrak{P}_{j}\right)^{-j}} \\
& \geq \lim _{j \rightarrow \infty}(1-\epsilon)^{-j} \\
& =\infty \text { on } A(R-\delta, R) .
\end{aligned}
$$

Similarly if there exists no sequence satisfying our Theorem 2.2 then

$$
\lim _{j \rightarrow \infty} \mathfrak{H}_{j}\left(\mathfrak{P}_{j}(r)\right)<\infty \text { for all } r \in[0, R),
$$

because if

$$
\lim _{j \rightarrow \infty} \mathfrak{H}_{j}\left(\mathfrak{P}_{j}(r)\right)=\infty \text { for } r_{0} \in[0, R)
$$

from

$$
\mathfrak{H}_{j}\left(\mathfrak{P}_{j}(r)\right) \geq \mathfrak{H}_{j}\left(\mathfrak{P}_{j}\left(r_{0}\right)\right) \text { for all }|x|=r \in\left[r_{0}, R\right)
$$

we deduce

$$
\begin{aligned}
-\lim _{j \rightarrow \infty} \Delta \mathfrak{P}_{j} & =\lim _{j \rightarrow \infty} \mathfrak{H}_{j}\left(\mathfrak{P}_{j}\right) \\
& \geq \lim _{j \rightarrow \infty} \mathfrak{H}_{j}\left(\mathfrak{P}_{j}\left(\mathrm{r}_{0}\right)\right)=\infty \text { on } A\left(r_{0}, R\right)
\end{aligned}
$$


Contradiction so 22 holds and we obtain that $\mathfrak{P} \in \mathrm{C}^{1}(\mathrm{~B}(0, \mathrm{R}))$, because using Theorem 9.11 page 235 in [18] we derive

$$
\begin{aligned}
\left\|\mathfrak{P}_{j}\right\|_{H^{2}, \mathfrak{p}\left(\Omega^{\prime \prime}\right)} & \leq \mathrm{C}\left(\mathrm{N}, \mathrm{p}, \Omega^{\prime}, \Omega^{\prime \prime}\right)\left\{\left\|\mathfrak{P}_{\mathfrak{j}}\right\|_{L^{p}\left(\Omega^{\prime}\right)}+\left\|\mathfrak{H}_{j}\left(\mathfrak{P}_{j}\right)\right\|_{L^{p}\left(\Omega^{\prime}\right)}\right\} \\
& \leq \mathrm{C}\left(\mathrm{N}, \mathrm{p}, \Omega^{\prime}, \Omega^{\prime \prime}\right)\left\{\|e\|_{L^{p}\left(\Omega^{\prime}\right)}+\left\|\lim _{j \rightarrow \infty} \mathfrak{H}_{j}\left(\mathfrak{P}_{j}\left(r_{3}\right)\right)\right\|_{L^{p}\left(\Omega^{\prime}\right)}\right\}
\end{aligned}
$$

where $\overline{\Omega^{\prime}} \subset \Omega^{\prime \prime}, \overline{\Omega^{\prime \prime}} \subset \mathrm{B}(0, R), \mathrm{p}>\mathrm{N}$ and $\mathrm{r}_{3}=\sup _{x \in \Omega^{\prime}}|x|$. According to Theorem 7.26 page 171 [18, we have the bounds

$$
\left\|\mathfrak{P}_{j}\right\|_{\mathrm{C}^{1,1-\frac{N}{\mathrm{p}}\left(\overline{\Omega^{\prime \prime}}\right)}} \leq\left\|\mathfrak{P}_{j}\right\|_{H^{2, p}\left(\Omega^{\prime \prime}\right)} \text {. }
$$

Therefore $\mathfrak{P} \in \mathrm{C}^{1}(\mathrm{~B}(0, \mathrm{R}))$ and $\mathfrak{P}_{j} \rightarrow \mathfrak{P}$ in $\mathrm{C}_{\text {loc }}^{1, \alpha}\left(\mathrm{B}(0, \mathrm{R})\right.$ for $0<\alpha<1-\frac{\mathrm{N}}{\mathrm{p}}$.

One more time if there exist no sequence satisfying the conclusions of Theorem 2.2 we imply $\mathfrak{P}:[0, R) \rightarrow[0, \infty)$ is a strictly nonincreasing radial function because if there exist $0 \leq r_{1}<r_{2}<R$ with $\mathfrak{P}\left(\mathrm{r}_{1}\right)=\mathfrak{P}\left(\mathrm{r}_{2}\right)$ and the fact of being $\mathfrak{P}$ nonincreasing implies $-\Delta \mathfrak{P}=0$ on the annulus $A\left(r_{1}, r_{2}\right)$. Using $\left\|\mathfrak{P}_{j}\right\|_{C^{1, \alpha}\left(A\left(r_{1}, r_{2}\right)\right.} \leq C$ and a nonnegative test function $\varphi$ with support contained in $A\left(r_{1}, r_{2}\right)$ :

$$
\begin{aligned}
0 & =\int_{\mathrm{A}\left(\mathrm{r}_{1}, \mathrm{r}_{2}\right)} \nabla \mathfrak{P} \cdot \nabla \varphi \mathrm{d} x \\
& =\lim _{j \rightarrow \infty} \int_{\mathrm{A}\left(\mathrm{r}_{1}, \mathrm{r}_{2}\right)} \nabla \mathfrak{P}_{j} \cdot \nabla \varphi \mathrm{d} x \\
& =\int_{\mathrm{A}\left(\mathrm{r}_{1}, \mathrm{r}_{2}\right)} \mathfrak{H}_{j}\left(\mathfrak{P}_{1}\right) \varphi \mathrm{d} x \\
& \geq \int_{\mathrm{A}\left(\mathrm{r}_{1}, \mathrm{r}_{2}\right)} \mathfrak{H}_{1}\left(\mathfrak{P}_{1}\right) \varphi \mathrm{d} x>0 .
\end{aligned}
$$

Contradiction.

So from the negation of the conclusion of Theorem 2.2 we derive that the function $\mathfrak{P}:[0, R) \rightarrow \mathbb{R}$ satisfies $\mathfrak{P} \in C^{1}(0, R), \mathfrak{P}$ is strictly nonincreasing in $(0, R)$ and $\lim _{r}>\mathbb{R} \mathfrak{P} \geq 1$ and therefore

$$
\mathfrak{P}(r)>1 \text { for all } r \in[0, R) \text {. }
$$

Finally we are ready to finish the proof of Theorem 2.2. Independently of the hypothesis in the reductio ad absurdum, there exists $0<r_{0}<R$ such that $\mathfrak{H}_{1}\left(\mathfrak{P}_{1}\left(r_{0}\right)\right)>\mathfrak{H}_{1}(1)=1$ and therefore using (iii) of Lemma 2.1

$$
1<\mathfrak{H}_{1}\left(\mathfrak{P}_{1}\left(r_{0}\right)\right) \leq \mathfrak{H}_{j}\left(\mathfrak{P}_{j}\left(r_{0}\right)\right) \text { for all } \mathfrak{j} \geq 1
$$

But $\lim _{j \rightarrow \infty} \mathfrak{P}_{j}\left(r_{0}\right)=\mathfrak{P}\left(r_{0}\right)>1$ and therefore for $j$ big enough $\mathfrak{P}_{j}\left(r_{0}\right)>1$ and

$$
\mathfrak{H}_{j}\left(\mathfrak{P}_{\mathfrak{j}}\left(\mathrm{r}_{\mathrm{O}}\right)\right)=\mathfrak{H}_{1}\left(\mathfrak{P}_{\mathfrak{j}}\left(\mathrm{r}_{0}\right)\right)<\mathfrak{H}_{1}(1)=1 .
$$

Therefore

$$
1<\mathfrak{H}_{1}\left(\mathfrak{P}_{1}\left(\mathrm{r}_{0}\right)\right) \leq \mathfrak{H}_{j}\left(\mathfrak{P}_{j}\left(\mathrm{r}_{0}\right)\right)<1,
$$

for $j$ big enough. In page 291 [11] it is stated that for every function $f \in \mathrm{C}^{\mathrm{m}, \alpha}(\Omega), 0<\alpha<1$, $\mathrm{m} \geq 1$ the solutions of Poisson's equation $\Delta \mathrm{u}=\mathrm{f}$ on $\Omega$ are of class $\mathrm{C}^{\mathrm{m}+2, \alpha}$ on $\Omega$, and so $\mathfrak{P}_{j}$ are of class $C^{\infty}(A(r, R))$. This end the proof. 
The next lemma is new

Lemma 2.3. The sequence $\left\{\mathfrak{H}_{j}\left(\mathfrak{P}_{j}\right)\right\}_{j=1}^{\infty}$ is unbounded in $\mathrm{C}_{\mathrm{loc}}^{\alpha}(\mathrm{A}(\mathrm{r}, \mathrm{R}))$.

Proof. In Theorem 4.6 page $60\left[18\right.$ it is stated that: let $\Omega$ be a domain in $\mathbb{R}^{N}$ and let $u \in C^{2}(\Omega)$, $\mathrm{f} \in \mathrm{C}^{\alpha}(\Omega)$ satisfy Poisson's equation $\Delta \mathrm{u}=\mathrm{f}$. Then $u \in \mathrm{C}^{2, \alpha}(\Omega)$ and for any two concentric balls $\mathrm{B}_{1}=\mathrm{B}\left(\mathrm{x}_{0}, \mathrm{R}\right), \mathrm{B}_{2}=\mathrm{B}\left(\mathrm{x}_{0}, 2 \mathrm{R}\right) \subset \subset \Omega$ we have

$$
|\mathrm{u}|_{2, \alpha ; \mathrm{B}_{1}}^{\prime} \leq \mathrm{C}\left(|\mathrm{u}|_{0 ; \mathrm{B}_{2}}+\mathrm{R}^{2}|\mathrm{f}|_{0, \alpha ; \mathrm{B}_{2}}^{\prime}\right) \text {. }
$$

Therefore $\lim _{j \rightarrow \infty}\left|\mathfrak{H}_{j}\left(\mathfrak{P}_{j}\right)\right|_{\hat{o}, \alpha ; \mathrm{B}_{2}}^{\prime}=\infty$.

\section{The construction of the sequence of functions $\mathfrak{F}_{j}$.}

In [19] it is stated that

[page XVIII, 19]] When we refer to a set F as a fractal, therefore, we will typically have the following in mind.

(i) F has a fine structure, i. e. detail on arbitray small scales.

(ii) $\mathrm{F}$ is too irregular to be described in traditional geometrical language, both locally and globally.

(iii) Often $\mathrm{F}$ has some form of self-similarity, perhaps approximate or statistical.

(iv) Usually, the 'fractal dimension' of $F$ (defined in some way) is greater than its topological dimension.

(v) In most cases of interest $F$ is defined in a very simple way, perhaps recursively.

Moreover

[Page XXII, [19] The highly intricate structure of the Julia set illustrated in figure 0.6 stems from the single quadratic function $f(z)=z^{2}+c$ for a suitable constant $c$. Although the set is not strictly self-similar in the sense that the Cantor's set and von Koch curve are, it is 'quasi-self-similar' in that arbitrarily small portions of the set can be magnified an distorted smoothly to coincide with a large part of the set. 
Let $[0, a]$ be a bounded interval in $\mathbb{R}$. Let us to consider, the infinite sequence of linear functions

$$
\begin{aligned}
\mathfrak{h}(x)=a-x, & \\
\mathfrak{h}_{0}(x)= & +1-x, \\
\mathfrak{h}_{1}(x)=a & +1+\frac{1}{2}-x, \\
& \ldots \\
\mathfrak{h}_{j}(x)=a & +\sum_{n=0}^{j} \frac{1}{2^{n}}-x .
\end{aligned}
$$

We introduce a infinite sequence of functions defined on $[0, a]$,

$$
\begin{gathered}
\mathfrak{p}(x)=\mathfrak{p}(x ; s ; k)=-t\left(x-\frac{s}{k} a\right)^{2}+\mathfrak{c}_{s} \text { for all } x \in\left[a \frac{s}{k}, a \frac{s+1}{k}\right], s=0,1,2, \ldots k-1, \\
\mathfrak{p}_{0}(x)=\mathfrak{p}_{0}\left(x ; s ; k_{0}\right)=-t_{0}\left(x-\frac{s}{k_{0}} a\right)^{2}+\mathfrak{c}_{0, s} \text { for all } x \in\left[a \frac{s}{k_{0}}, a \frac{s+1}{k_{0}}\right], s=0,1,2, \ldots k_{0}-1, \\
\mathfrak{p}_{1}(x)=\mathfrak{p}_{1}\left(x ; s ; k_{1}\right)=-t_{1}\left(x-\frac{s}{k_{1}} a\right)^{2}+\mathfrak{c}_{1, s} \text { for all } x \in\left[a \frac{s}{k_{1}}, a \frac{s+1}{k_{1}}\right], s=0,1,2, \ldots k_{1}-1, \\
\ldots, \\
\mathfrak{p}_{j}(x)=\mathfrak{p}_{j}\left(x ; s ; k_{j}\right)=-t_{j}\left(x-\frac{s}{k_{j}} a\right)^{2}+\mathfrak{c}_{j, s} \text { for all } x \in\left[a \frac{s}{k_{j}}, a \frac{s+1}{k_{j}}\right], s=0,1,2, \ldots k_{j}-1 .
\end{gathered}
$$

Where the sequence

$$
\begin{array}{r}
\mathfrak{c}_{0}=1, \\
\mathfrak{c}_{0,0}=1+1, \\
\mathfrak{c}_{1,0}=1+1+\frac{1}{2}, \\
\ldots, \\
\mathfrak{c}_{j, 0}=1+\sum_{s=0}^{j} \frac{1}{2^{j}} .
\end{array}
$$

satisfies

$$
\begin{array}{r}
\mathfrak{c}_{s}=\mathfrak{p}\left(\frac{s}{k} ; s ; k\right) \text { for all } s=1, \ldots, k-1, \\
\mathfrak{c}_{0, s}=\mathfrak{p}_{0}\left(\frac{s}{k} ; s ; k_{0}\right) \text { for all } s=1, \ldots, k_{0}-1, \\
\mathfrak{c}_{1, s}=\mathfrak{p}_{1}\left(\frac{s}{k} ; s ; k_{1}\right) \text { for all } s=1, \ldots, k_{1}-1, \\
\ldots, \\
\mathfrak{c}_{j, s}=\mathfrak{p}_{j}\left(\frac{s}{k_{j}} ; s ; k_{j}\right) \text { for all } s=1, \ldots, k_{j}-1 .
\end{array}
$$


We have the association:

$$
\begin{array}{r}
\left\{\mathfrak{c}_{s}\right\}_{s=0}^{\infty} \longleftrightarrow \mathfrak{h} \longleftrightarrow \mathfrak{p}, \\
\left\{\mathfrak{c}_{0, s}\right\}_{s=0}^{\infty} \longleftrightarrow \mathfrak{h}_{0} \longleftrightarrow \mathfrak{p}_{0}, \\
\left\{\mathfrak{c}_{1, s}\right\}_{s=0}^{\infty} \longleftrightarrow \mathfrak{h}_{1} \longleftrightarrow \mathfrak{p}_{1}, \\
\ldots, \\
\left\{\mathfrak{c}_{j, s}\right\}_{s=0}^{\infty} \longleftrightarrow \mathfrak{h}_{j} \longleftrightarrow \mathfrak{p}_{j} .
\end{array}
$$

The choice of the sequence of non negative numbers $k \cup\left\{k_{j}\right\}_{j=1}^{\infty}$ determines $t \cup\left\{t_{j}\right\}_{j=1}^{\infty}$. We keep $k$ such that the equation

$$
\mathfrak{p}(x ; 0, k)=\mathfrak{h}_{0}(x),
$$

has no solutions. Similarly we divide this first interval $\left[0, \frac{a}{k}\right]$ in $\mathfrak{k}_{0}$ intervals and setting $k_{0}=k_{k_{0}}$ such that the equation

$$
\mathfrak{p}_{0}(x ; 0, k)=\mathfrak{h}_{1}(x)
$$

has no solution. Now we complete the procedure by induction. Therefore

$$
\lim _{j \rightarrow \infty} t_{j}=\infty .
$$

It is follow that the non decreasing, bounded, sequence of functions $\{\mathfrak{p}\}_{\mathfrak{j}=0}^{\infty}$ defined on $[0, a]$ has second derivative defined almost everywhere and $\mathfrak{p}_{j}^{\prime \prime}(x)=-2 t_{j}$.

Using the Rolle's Theorem for the functions $\mathfrak{p}_{j}\left(\cdot ; s ; \mathfrak{k}_{\mathfrak{j}}\right), \mathfrak{h}_{\mathfrak{j}}(\cdot)$ in the interval $\left[\frac{s a}{k_{j}}, \frac{(s+1) \mathfrak{a}}{k_{j}}\right]$, we deduce the existence of $x_{j, s} \in\left(\frac{s a}{k_{j}}, \frac{(s+1) a}{k_{j}}\right)$ such that

$$
\mathfrak{p}_{j}^{\prime}\left(x_{\mathfrak{j}, s} ; s ; \mathfrak{k}_{\mathfrak{j}}\right)=\mathfrak{h}_{j}^{\prime}\left(x_{\mathfrak{j}, s}\right)=-1,
$$

where

$$
x_{j, s}=\frac{1}{2 t_{j}}+\frac{s a}{k_{j}} .
$$

Similarly for

$$
\tilde{x}_{j, s}=\frac{1}{20 t_{j}}+\frac{s a}{k_{j}}
$$

we have

$$
\mathfrak{p}_{j}^{\prime}\left(\tilde{x}_{j, s} ; ; ; k_{j}\right)=-\frac{1}{10} .
$$

For $j$ big enough, let us to consider the sequence of intervals $\left(\frac{s a}{k_{j}}-\delta_{j}, \frac{s a}{k_{j}}+\delta_{j}\right) \subset[0, a]$ for $s=$ $1,2, \ldots, k_{j}-1$ where

$$
\delta_{j}=\min \left\{\frac{1}{200 t_{j}}, \frac{a}{k_{j} j^{2}}, \frac{\frac{a}{k_{j}}-\frac{1}{2 t_{j}}}{10}\right\}
$$


By smoothing each $\mathfrak{p}_{j}$ on $\left(\frac{s a}{k_{j}}-\delta_{j}, \frac{s a}{k_{j}}+\delta_{j}\right)$, we obtain the desired sequence $\left\{\mathfrak{F}_{j}\right\}_{j=1}^{\infty} \in C^{\infty}[a, b]$. We point that $\mathrm{C}^{\infty}$ extension is a nontrivial task, see for example page 136 [18] or the Nikolskii's extension method page 69 [29] and the Calderon's extension method page 72 [29]. Therefore we give a full description of our procedure. For the smoothing method we use the functions

$$
S(x ; \mathfrak{a} ; \mathfrak{b})=x-\int_{0}^{x} g(t) d t
$$

where $g(x)=0$ for $x<\mathfrak{a}-\delta, g(x)=1$ for $\mathfrak{a}<x<\mathfrak{b}, g(x)=0$ for $x>\mathfrak{b}+\delta, 0<\delta \in \mathbb{R}$, and $g \in C^{\infty}(\mathbb{R})$ (see the $C^{\infty}$ Urysohn Lemma page 245 , 14]). It it follows that $S(x ; \mathfrak{a} ; \mathfrak{b})=x$ for $x<\mathfrak{a}-\delta, S(x ; \mathfrak{a} ; \mathfrak{b})=x-\mathfrak{c}$ for $x>\mathfrak{b}+\delta$ and $S^{\prime}(x ; \mathfrak{a} ; \mathfrak{b}) \geq 0$. Taking a suitable composition with functions $S(\cdot ; \mathfrak{a} ; \mathfrak{b})$ we accomplish with the smoothing procedure and moreover $\mathfrak{F}_{\mathfrak{j}}^{\prime}(x) \leq 0$. The sequence $\left\{\Delta \mathfrak{F}_{j}\right\}_{j=1}^{\infty}$ converges in measure to $-\infty$.

Note that the set of numbers $\left\{x_{j, s}, \tilde{x}_{j, s}\right\}$ are numerable and dense in $[0, a]$. Therefore by 42 and 45 we imply if $\mathfrak{F}_{\mathfrak{j}}\left(x_{1}, x_{2}, \ldots, x_{N}\right)=\mathfrak{F}_{j}\left(x_{1}\right)$ then

$$
\lim _{j \rightarrow \infty}\left\|\mathfrak{F}_{j}\right\|_{C^{1, \alpha}(\overline{B(x, r)})}=\infty,
$$

for any ball $\mathrm{B}(x, r)$ with center at $x$ with radius $r$ and $\overline{\mathrm{B}(x, r)} \subset \Omega$. It follows that

$$
\lim _{j \rightarrow \infty}\left\|\mathfrak{F}_{j}\right\|_{C^{2, \alpha}(\overline{B(x, r)})}=\infty .
$$

Let us to consider the function

$$
I_{A_{j}}(x)= \begin{cases}1 & \text { if } x \in A_{j}, \\ 0 & \text { if } x \notin A_{j},\end{cases}
$$

where $A_{j}=\left\{x \in\left(\frac{s a}{k_{j}}+\delta_{j}, \frac{(s+1) a}{k_{j}}-\delta_{j}\right) \mid s=0,1,2, \ldots k_{j}-1\right\}$. Therefore

$$
\begin{aligned}
\int_{0}^{a} \mathfrak{F}_{j}^{\prime \prime} \mathrm{I}_{A_{j}} \mathrm{dx} & =\mathrm{t}_{j} \sum_{s=0}^{k_{j}-1}\left(\frac{\mathrm{a}}{\mathrm{k}_{j}}-2 \delta_{j}\right) \\
& =\mathrm{t}_{j} \mathrm{a}-2 \sum_{s=0}^{k_{j}-1} \delta_{j} \\
& \geq \mathrm{t}_{j} a-2 \frac{\mathrm{a}}{\mathfrak{j}^{2}} .
\end{aligned}
$$

So $\lim _{j \rightarrow \infty} \int_{0}^{a} \mathfrak{F}_{j}^{\prime \prime} \mathrm{I}_{A_{j}} \mathrm{~d} x=\infty$ and

$$
\lim _{j \rightarrow \infty}\left\|\mathfrak{F}_{j}^{\prime \prime}\right\|_{L^{p}[0, a]}=\infty \text { for all } 1 \leq p \leq \infty .
$$

\section{A primer analysis.}

\subsection{A new kind of ill-posed problem.}

The concept of a well-posed problem of mathematical physics was introduced by J. Hadamard. The solution of any quantitative problem usually ends in a equation $z=R(u)$ where $u$ is the 
initial data and $z$ is the solution, $\mathrm{R}: \mathrm{U} \rightarrow \mathrm{Z}, \mathrm{U}$ and $\mathrm{Z}$ are metric spaces with distances $\rho_{\mathrm{u}}$ and $\rho_{Z}$ respectively. The problem of determining the solution $z$ in the space $Z$ from the initial data $u$ in the space $U$ is said to be well-posed on the pair of metric space $(Z, U)$ if the following three conditions are satisfied:

(i) For every element $\mathfrak{u} \in \mathrm{U}$ there exists a solution $z$ in the space $\mathbf{Z}$.

(ii) The solution is unique.

(iii) For every positive number $\epsilon>0$ there exists a positive number $\delta$ such that $\rho_{\mathrm{u}}\left(\mathfrak{u}_{1}, \mathfrak{u}_{2}\right) \leq \delta$ implies $\rho_{Z}\left(z_{1}, z_{2}\right) \leq \epsilon$, where $z_{1}=S\left(u_{1}\right), z_{2}=S\left(u_{2}\right)$.

Problems that do not satisfy them are said ill-posed. The sequence $\left\{\mathfrak{P}_{j}\right\}_{j=1}^{\infty}$ is a new kind of illposed problem related to Sobolev's spaces or even for the Laplacian operator in the the context of distributions.

Given $\Omega$ a bounded domain in $\mathbb{R}^{N}$ and $u \in W^{1,1}(\Omega)$ whose laplacian is a bounded measure $\mu$ on $\Omega$, we call normal derivative in the sense of distributions of $u$ on $\partial \Omega$ the distribution $\mathfrak{v}_{1}$ defined on $\mathbb{R}^{N}$ by

$$
\left\langle\mathfrak{v}_{1}, \varphi\right\rangle=\int_{\Omega} \varphi \mathrm{d} \mu+\int_{\Omega} \nabla \varphi \cdot \nabla \mathrm{ud} x, \varphi \in \mathscr{D}\left(\mathbb{R}^{\mathrm{N}}\right)
$$

The distribution $\mathfrak{v}_{1}$ defined by 52 is of compact support in $\partial \Omega$, if $\operatorname{supp} \varphi \cap \partial \Omega=\emptyset$ then $\varphi \mathrm{I}_{\Omega}$ and $\nabla\left(\varphi \mathrm{I}_{\Omega}\right)=(\nabla \varphi) \mathrm{I}_{\Omega}$. Then by the definition of Laplacian in the sense of distributions

$$
\begin{aligned}
\int_{\Omega} \varphi \mathrm{d} \mu & =\left\langle\Delta \mathrm{u}, \varphi \mathrm{I}_{\Omega}\right\rangle \\
& =-\int \nabla\left(\varphi \mathrm{I}_{\Omega}\right) \cdot \nabla \mathrm{ud} x \\
& =-\int_{\Omega} \nabla \varphi \cdot \nabla \mathrm{udx} .
\end{aligned}
$$

Proposition 4.1 (page 500 [11]). Let $\Omega$ be a regular bounded open set with boundary of class $\mathrm{W}^{2, \infty}, \mu$ a bounded Radon measure and $\mathfrak{v}_{1}$ a Radon measure on $\partial \Omega$. Let us to consider the Neumann problem

$$
u \in W^{1,1}(\Omega), \Delta u=\mu \text { in } \mathscr{D}^{\prime}(\Omega),
$$

$\mathfrak{v}_{1}$ is the normal derivative on $\partial \Omega$ of $\mathrm{u}$.

(i) There exists a solution of 5455 if and only if $\int_{\partial \Omega} d \mathfrak{v}_{1}=\int_{\Omega} d \mu$.

(ii) If $\int_{\partial \Omega} d \mathfrak{v}_{1}=\int_{\Omega} \mathrm{d} \mu$ then the solution of 54,55 is defined to whitin an additive constant and $\mathrm{u} \in \mathrm{W}^{1, \mathrm{p}}(\Omega)$ for all $1 \leq \mathrm{p}<\frac{\mathrm{N}}{\mathrm{N}-1}$.

Our sequence $\left\{\Delta \mathfrak{P}_{j}\right\}_{j=1}^{\infty}$ is non bounded in $L^{\infty}(\Omega)$ therefore the limit $\mathfrak{P}$ is outside of the scope of application of Proposition 4.1 . 


\subsubsection{Green's identities.}

We show the nature of the discontinuity of the the sequence $\left\{\mathfrak{P}_{j}\right\}_{j=1}^{\infty}$ in Sobolev's spaces and in the context of distribution theory. From (page 17 [18, or [6])

$$
\int_{\Omega}(\Delta \mathrm{u}) v \mathrm{~d} x=\int_{\partial \Omega} \frac{\partial u}{\partial \mathrm{n}} v \mathrm{~d} \gamma-\int_{\Omega} \nabla \mathrm{u} \cdot \nabla v \mathrm{~d} x \text { for all } \mathrm{u} \in \mathrm{C}^{2}(\bar{\Omega}), \text { for all } v \in \mathrm{C}^{1}(\bar{\Omega}),
$$

we calculate

$$
\lim _{j \rightarrow \infty} \int_{\Omega} \nabla \mathfrak{P}_{j} \cdot \nabla v \mathrm{~d} x=\infty \text { for all non negative } v \in \mathrm{C}^{1}(\bar{\Omega}) \text { with } v=0 \text { on } \partial \Omega .
$$

Taking $v \equiv 1$ in 56 , we derive

$$
\lim _{j \rightarrow \infty} \int_{\partial \Omega} \frac{\partial \mathfrak{P}_{j}}{\partial \mathrm{n}} \mathrm{d} \gamma=-\infty
$$

Now we use the second Green's identity

$$
\int_{\Omega}(v \Delta u-u \Delta v) d x=\int_{\partial \Omega}\left(v \frac{\partial u}{\partial n}-u \frac{\partial v}{\partial n}\right) d \gamma \text { for all } u, v \in C^{2}(\bar{\Omega})
$$

it follows

$$
\lim _{j \rightarrow \infty} \int_{\partial \Omega}\left(v \frac{\partial \mathfrak{P}_{j}}{\partial n}-\mathfrak{P}_{j} \frac{\partial v}{\partial n}\right) d \gamma=-\infty \text { for all nonnegative } v \in \mathrm{C}^{2}(\bar{\Omega}) .
$$

Therefore for non negative $v \in \mathrm{C}^{2}(\bar{\Omega})$ with $v=0$ on $\partial \Omega$, we deduce

$$
\begin{array}{r}
\int_{\Omega}\left((v+\epsilon) \Delta \mathfrak{P}_{j}-\mathfrak{P}_{j} \Delta v\right) \mathrm{d} x=\epsilon \int_{\partial \Omega} \frac{\partial \mathfrak{P}_{j}}{\partial \mathrm{n}} \mathrm{d} \gamma-\int_{\partial \Omega} \mathfrak{P}_{j} \frac{\partial v}{\partial \mathrm{n}} \mathrm{d} \gamma \\
\int_{\Omega}\left(v \Delta \mathfrak{P}_{j}-\mathfrak{P}_{j} \Delta v\right) \mathrm{d} x=-\int_{\partial \Omega} \mathfrak{P}_{j} \frac{\partial v}{\partial \mathrm{n}} \mathrm{d} \gamma
\end{array}
$$

and letting $j \rightarrow \infty$ we demonstrate the discontinuity at infinitum of the functional 3 ,

Theorem 4.2 (Theorem 4.11 page $85[29]$ ). Let $\Omega \in \mathbb{R}^{N}$ a bounded domain with smooth boundary; if $1 \leq \mathrm{p}<\mathrm{N}$ put $\frac{1}{\mathrm{q}}=\frac{1}{\mathrm{p}}-\frac{1}{\mathrm{~N}-1} \frac{\mathrm{p}-1}{\mathrm{p}}$; if $\mathrm{p}=\mathrm{N}$, put $\mathrm{q} \geq 1$. There exists a unique mapping $\mathrm{Z} \in\left[\mathrm{W}^{2, \mathfrak{p}}(\Omega) \rightarrow \mathrm{W}^{1, \mathrm{q}}(\partial \Omega)\right]$ such that $\mathrm{u} \in \mathrm{C}^{\infty}(\bar{\Omega}) \Longrightarrow \mathrm{Zu}=\mathrm{u}$.

The second Green's identity 59 is valid on $\mathrm{W}^{2,2}(\Omega)$, it follows from 58 and Theorem 4.2 that our sequence $\left\{\mathfrak{P}_{j}\right\}_{\mathfrak{j}=1}^{\infty}$ is unbounded in $W^{1,2 \frac{N-1}{N-2}}(\partial \Omega)$. From 57 we imply

$$
\lim _{j \rightarrow \infty}\left\|\mathfrak{P}_{j}\right\|_{W^{1, p}(\Omega)}=\infty \text { for all } 1 \leq p \leq \infty
$$

and

$$
\lim _{j \rightarrow \infty}\left\|\mathfrak{P}_{j}\right\|_{W^{2, p}(\Omega)}=\infty \text { for all } 1 \leq p \leq \infty .
$$

Therefore the sequence is unbounded in the domain of definition of the trace operator stated in Theorem 4.2. Similar considerations are implied easily from 
Theorem 4.3 (page 5 [29]). Let $\Omega$ be a bounded domain with lipschitzian boundary. Then there exists a uniquely defined, linear an continuous mapping $T: W^{k, 2}(\Omega) \rightarrow \mathrm{L}^{2}(\partial \Omega)$ such that for $x \in \partial \Omega$ and $\mathfrak{v} \in \mathrm{C}^{\infty}(\Omega)$, it is defined by $\mathrm{T}(\mathfrak{v})(\mathrm{x})=\mathfrak{v}(\mathrm{x})$

Theorem 4.4 (page $135[36]$ ). We consider a solution $\mathfrak{u}=\mathfrak{u}(x) \in \mathrm{C}^{2}(\Omega)$ of Poisson's differential equation $\Delta \mathrm{u}(\mathrm{x})=\mathrm{f}(\mathrm{x}), \mathrm{x} \in \Omega$ in the domain $\Omega \subset \mathbb{R}^{\mathrm{N}}, \mathrm{N} \geq 3$. For each ball $\mathrm{B}_{\mathrm{R}}(\mathrm{a}) \subset \subset \Omega$ then we have the identity

$$
u(a)=\frac{1}{R^{N-1} \omega_{N}} \int_{|x-a|=R} u(x) d \sigma-\frac{1}{(N-2) \omega_{N}} \int_{|x-a| \leq R}\left(|x-a|^{2-N}-R^{2-N}\right) f(x) d x
$$

The same discontinuity at infinitum appears in the context of singular phenomena in nonlinear elliptic problems [1, 2, 9, 17, 34.

$$
\begin{array}{r}
\int_{\Omega}\left((v+\epsilon) \Delta \mathfrak{U}_{j}-\mathfrak{U}_{j} \Delta v\right) \mathrm{d} x=\epsilon \int_{\partial \Omega} \frac{\partial \mathfrak{U}_{j}}{\partial \mathrm{n}} \mathrm{d} \gamma-\int_{\partial \Omega} \mathfrak{U}_{j} \frac{\partial v}{\partial \mathrm{n}} \mathrm{d} \gamma, \\
\int_{\Omega}\left(v \Delta \mathfrak{U}_{j}-\mathfrak{U}_{j} \Delta v\right) \mathrm{d} x=-\int_{\partial \Omega} \mathfrak{U}_{j} \frac{\partial v}{\partial \mathrm{n}} \mathrm{d} \gamma,
\end{array}
$$

where $\mathfrak{U}_{j}$ solves the problem

$$
\begin{aligned}
-\Delta \mathfrak{U}_{j} & =\mathfrak{U}_{j}^{-\gamma} \text { in } \Omega, \\
\mathfrak{U}_{j} & =\frac{1}{\mathfrak{j}} \text { on } \partial \Omega,
\end{aligned}
$$

with $\gamma>1$. Moreover $\lim _{j \rightarrow \infty} \mathfrak{U}_{j}=\mathfrak{U} \in \mathrm{C}^{2}(\Omega) \cap \mathrm{C}^{0}(\bar{\Omega})$ and $\int_{\Omega} \mathfrak{U}^{-\gamma} \mathrm{d} x=\infty$, showing the same kind of discontinuity at infinitum. This discontinuity property is an interesting example in the Friedrichs method of extension of semibounded operators to self-adjoint operators (page 228 [4, see also page 205 [24]).

\subsubsection{Integration by parts.}

In the one dimension if $u, v \in W^{1, p}(I)$ with $1 \leq p \leq \infty$, then $\int_{x}^{y} u^{\prime} v=u(x) v(x)-u(y) v(x)-\int_{y}^{x} u v^{\prime}$ for all $x, y \in \overline{\mathrm{I}}$ but even if a distribution has second distributional derivative the integration by parts is not true. For example for $f_{h}=\int_{0}^{x} \frac{1}{h} I_{[-h, 0]}(t) d t$, we have:

$$
\begin{aligned}
\int_{x}^{y}\left[f_{h}^{\prime}\right] \varphi^{\prime} & =\left[f_{h}^{\prime}(y)\right] \varphi(y)-\left[f_{h}^{\prime}(x)\right] \varphi(x)-\int_{x}^{y}\left[f_{h}^{\prime \prime}\right] \varphi=0 & & \text { for all } \varphi \in C_{0}^{\infty}(x, y) \\
\int_{x}^{y}\left[f_{h}^{\prime}\right] \varphi^{\prime} & =\left[f_{h}(y)\right] \varphi^{\prime}(y)-\left[f_{h}(x)\right] \varphi^{\prime}(x)-\int_{x}^{y}\left[f_{h}\right] \varphi^{\prime \prime} & & \\
& =\frac{\varphi^{\prime}(-h)-\varphi^{\prime}(0)}{-h} & & \text { for all } \varphi \in C_{0}^{\infty}(x, y) .
\end{aligned}
$$

Taking a radial $\varphi \in \mathscr{D}(A(r, R))$ we get

$$
\begin{aligned}
\int_{A(r, R)} \nabla \mathfrak{P}_{j} \cdot \nabla \varphi \mathrm{d} x & =\int_{A(r, R)} \frac{\partial \mathfrak{P}_{j}}{\partial \mathfrak{r}} \frac{x_{i}}{\mathfrak{r}} \frac{\varphi}{\partial \mathfrak{r}} \frac{x_{i}}{\mathfrak{r}} \mathrm{dx} \\
& =\int_{A(r, R)} \frac{\partial \mathfrak{P}_{j}}{\partial \mathfrak{r}} \frac{\partial \varphi}{\partial \mathfrak{r}} \mathfrak{r} d x \\
& =\int_{r}^{R} \mathfrak{r}^{N-1} d \mathfrak{d} \int_{S^{N-1}} \frac{\partial \mathfrak{P}_{j}}{\partial \mathfrak{r}} \frac{\partial \varphi}{\partial \mathfrak{r}} \mathfrak{r} \mathrm{d} \omega \\
& =\int_{r}^{R} \frac{\partial \mathfrak{P}_{j}}{\partial \mathfrak{r}} \frac{\partial \varphi}{\partial \mathfrak{r}} \mathfrak{r}^{N} d \mathfrak{r} \int_{S^{N-1}} \mathrm{~d} \omega .
\end{aligned}
$$


So

$$
\lim _{j \rightarrow \infty} \int_{r}^{R} \frac{\partial \mathfrak{P}_{j}}{\partial \mathfrak{r}} \frac{\partial \varphi}{\partial \mathfrak{r}} d \mathfrak{r}=\infty
$$

and the integration by parts rule not hold in the limit because

$$
\int_{r}^{R} \frac{\partial \mathfrak{P}_{j}}{\partial \mathfrak{r}} \frac{\partial \varphi}{\partial \mathfrak{r}} d \mathfrak{r}=\mathfrak{P}_{j}(R) \frac{\partial \varphi}{\partial \mathfrak{r}}(R)-\mathfrak{P}_{j}(r) \frac{\partial \varphi}{\partial \mathfrak{r}}(r)-\int_{r}^{R} \mathfrak{P}_{j} \frac{\partial^{2} \varphi}{\partial \mathfrak{r}^{2}} d \mathfrak{r}
$$

Also the Cantor's function not satisfies the integration by part rule (this function is monotone and it has zero derivative almost everywhere).

\subsubsection{A detour with monotone functions.}

Let $\Omega$ be a domain in $\mathbb{R}^{N}, \mathrm{P}$ a line verifying $\mathrm{P} \cap \Omega$ is a nonempty set. A function defined almost everywhere in $\Omega$ is said absolutely continuous on the line $P$ if it is continuous on each closed interval of $\mathrm{P} \cap \Omega$.

Theorem 4.5 (page $55[29]$ ). Suppose $u \in \mathrm{L}_{\mathrm{loc}^{1}}(\Omega)$ and $\frac{\partial \mathrm{u}}{\partial \mathrm{x}_{\mathrm{i}}} \in \mathrm{L}^{\mathrm{p}}(\Omega), \mathrm{p} \geq 1$. This function changed on a set of measure zero is absolutely continuous on almost all lines parallel to axis $x_{i}$. Let us denote by $\left[\frac{\partial \mathrm{u}}{\partial x_{i}}\right]$ the usual derivative and by $\frac{\partial u}{\partial x_{i}}$ the distribution derivative. Then we have almost everywhere $\left[\frac{\partial \mathrm{u}}{\partial \mathrm{x}_{\mathrm{i}}}\right]=\frac{\partial \mathrm{u}}{\partial x_{i}}$. Conversely, if $\mathrm{u} \in \mathrm{L}_{\mathrm{loc}_{\mathrm{oc}}}^{1}(\Omega)$ is absolutely continuous on almost all lines parallel to the axis $x_{i}$ with $\left[\frac{\partial u}{\partial x_{i}}\right] \in L^{p}(\Omega)$, then we have $\frac{\partial u}{\partial x_{i}}=\left[\frac{\partial u}{\partial x_{i}}\right]$.

By the Lebesgue's Differentiation Theorem the function $\mathfrak{P}$ has derivative almost everywhere with respect the radius.

$$
\begin{aligned}
\operatorname{Var}_{j} & =\sup _{r \leq r_{0}<r_{1}<\cdots<r_{n} \leq R}\left\{\sum_{i=1}^{n}\left|\mathfrak{P}_{j}\left(r_{i}\right)-\mathfrak{P}_{j}\left(r_{j-1}\right)\right|\right\} & & (\text { page 39 [22]) } \\
& \left.=\int_{r}^{R} \mid\left[\frac{\mathrm{d} \mathfrak{P}_{j}}{\mathrm{dr}}\right]\right) \mid \mathrm{dr} & & (\text { page 41 [22]) } \\
& =\sup _{[\mathrm{r}, \mathrm{R}]} \mathfrak{P}_{j}-\inf _{[\mathrm{r}, \mathrm{R}]} \mathfrak{P}_{j} & & \text { (page 43 [22]) } \\
& \leq \mathrm{C} & & \text { (Lemma 2.1]iv). }
\end{aligned}
$$

Moreover from page $62[22$

$$
\operatorname{Var} \mathfrak{P} \leq \liminf _{n \rightarrow \infty} \operatorname{Var}_{j}
$$

We infer

$$
\left\|\left[\frac{\mathrm{d} \mathfrak{P}}{\mathrm{d} \mathfrak{r}}\right]\right\|_{L^{1}[r, R]} \leq\left\|\left[\frac{\mathrm{d} \mathfrak{P}_{\mathfrak{j}}}{\mathrm{dr}}\right]\right\|_{L^{1}[r, R]} \leq \mathrm{Cte} .
$$

From

$$
\mathfrak{P}_{j}^{\prime \prime}(\mathfrak{r})+\frac{N-1}{\mathfrak{r}} \mathfrak{P}_{j}^{\prime}(\mathfrak{r}) \leq C_{j} \text { on } B(r, R) \text { with } \lim _{j \rightarrow \infty} C_{j}=-\infty
$$

we derive

$$
\lim _{j \rightarrow \infty}\left\|\frac{\partial^{2} \mathfrak{P}_{j}}{\partial \mathfrak{r}^{2}}\right\|_{L^{p}(B(r, R))}=\infty
$$


$1 \leq p \leq \infty$. Similarly

$$
\lim _{j \rightarrow \infty}\left\|\mathfrak{F}^{\prime \prime}\right\|_{L^{p}[0, a]}=\infty
$$

for all $1 \leq p \leq \infty$ and

$$
\left\|\left[\frac{\mathrm{d} \mathfrak{F}}{\mathrm{d} x}\right]\right\|_{L^{1}[0, \mathrm{a}]} \leq\left\|\left[\frac{\mathrm{d} \mathfrak{F}_{j}}{\mathrm{dx}}\right]\right\|_{L^{1}[0, \mathrm{a}]} \leq \text { Cte. }
$$

It follows that both sequences $\left\{\mathfrak{P}_{j}\right\}_{j=1}^{\infty}$ and $\left\{\mathfrak{F}_{j}\right\}_{j=1}^{\infty}$ are ill-posed in Sobolev's spaces. This anomalous behaviour is also present in the sequence $f_{h}(x)=\int_{0}^{x} \frac{1}{h} I_{[-h, 0]}(t) d t$, where $\int_{-1}^{1}\left|\left[\frac{d f_{h}}{d t}\right]\right| d t=1$ for all $h>0$ and $\lim _{h \rightarrow 0^{+}}\left\|\left[\frac{d f_{h}}{d t}\right]\right\|_{L^{p}[-1,1]}=\infty$ for all $1<p \leq \infty$. Moreover for all nonnegative test function $\varphi \in \mathrm{C}_{0}^{\infty}(-1,1)$, we have

$$
\lim _{h \rightarrow 0^{+}} \int_{-1}^{1}\left[f_{h}^{\prime}(x)\right] \varphi^{\prime}(x) d x=\lim _{h \rightarrow 0^{+}} \int_{-h}^{0} \frac{\varphi^{\prime}(x)}{h} d x=\lim _{h \rightarrow 0^{+}} \frac{\varphi(-h)-\varphi(0)}{-h}=(-1)\left(D \delta_{0}\right) \varphi .
$$

Therefore if we define the distribution $\Lambda_{h}(\varphi)=\int_{-1}^{1}\left[f_{h}^{\prime}(x)\right] \varphi^{\prime}(x) d x$ then $\lim _{h \rightarrow 0^{+}} \Lambda_{h}=-\left(D \delta_{0}\right)$ where $\mathrm{D} \delta_{0}$ is the distributional derivative of Dirac's $\delta$ distribution and it is well known that distribution has not weak derivative. The space of functions of pointwise bounded variation admits discontinuous functions and therefore both topologies on the same set $C^{\infty}(r, R)$ produce completely different objects in metrics and associated functionals. Our functions The sequence $\left\{\mathfrak{P}_{j}\right\}_{j=1}^{\infty}$ is bounded in $W^{1,1}(A(r, R))$ but is it not ensured the strong or weak convergence. The function $\mathfrak{P}$ has derivative $\left[\frac{\partial \mathfrak{P}}{\partial \mathfrak{r}}\right]$ almost everywhere on $(r, R)$ because is a monotone function and moreover we have $\mathfrak{P}=\mathfrak{P}_{A C}+\mathfrak{P}_{C}+\mathfrak{P}_{j}$, where $\mathfrak{P}_{A C}$ is an absolutely continuous function, $\mathfrak{P}_{j}$ is continuous and singular, and $\mathfrak{P}_{\mathrm{J}}$ is the jump function of $\mathfrak{P}$.

Theorem 4.6 (page 3 22]). Let $\mathrm{I} \subset \mathbb{R}$ be an interval and let $\mathrm{u}: \mathrm{I} \rightarrow \mathbb{R}$ be a monotone function. Then $\mathrm{u}$ has as most countable many discontinuity points. Conversely, given a countable set $\mathrm{E} \subset \mathbb{R}$, there exists a monotone function $\mathrm{u}: \mathbb{R} \rightarrow \mathbb{R}$ whose set of discontinuity points is exactly $\mathrm{E}$.

So by Theorem 4.5 the function $u$ has derivative $\left[\mathfrak{u}^{\prime}\right]$ but no weak derivative if for example $E$ is dense on $\mathrm{I}$.

\subsection{The solid mean value.}

Despite the difficulty posed by the discontinuity of Green's identities on the sequence $\left\{\mathfrak{P}_{j}\right\}_{\mathfrak{j}=1}^{\infty}$ we can obtain several properties.

If a function $u$ is absolutely continuous on the interval $(a, b)$ page $225[22$, then

$$
u(x)-\frac{1}{b-a} \int_{a}^{b} u(t) d t=\frac{1}{b-a}\left[\int_{a}^{x}(t-a) u^{\prime}(t) d t-\int_{x}^{b}(b-t) u^{\prime}(t) d t\right] .
$$

Using the Lebesgue's Dominated Convergence Theorem, we obtain a one dimensional solid mean average identity

$$
\mathfrak{F}(x)-\frac{1}{b-a} \int_{a}^{b} \mathfrak{F}(t) d t=\lim _{j \rightarrow \infty}\left\{\frac{1}{b-a}\left[\int_{a}^{x}(t-a) \mathfrak{F}_{j}^{\prime}(t) d t-\int_{x}^{b}(b-t) \mathfrak{F}_{j}^{\prime}(t) d t\right]\right\} .
$$


Now we recall the extension to Poisson's equation of the solid mean value for Laplace's equation in [18] Chapter 4: Let $v \in C^{2}(\Omega) \cap C^{0}(\bar{\Omega})$ satisfy $-\Delta v=f$ then for any ball $B=B_{R}(y)$, we have

$$
v(y)=\frac{1}{|B|} \int_{B} v d x+\frac{1}{N \omega_{N}} \int_{B} f(x) \Theta(r, R) d x, r=|x-y|,
$$

where

$$
\Theta(r, R)=\frac{1}{N-2}\left(r^{2-N}-R^{2-N}\right)-\frac{1}{2 R^{N}}\left(R^{2}-r^{2}\right)
$$

for $N>2$ and

$$
\Theta(r, R)=\log \left(\frac{R}{r}\right)-\frac{1}{2}\left(1-\frac{r^{2}}{R^{2}}\right)
$$

for $N=2$, where $\omega_{N}$ is the volume of the unit ball in $\mathbb{R}^{N}$. We deduce that if $\mathfrak{P}(y)=\lim _{\mathfrak{j} \rightarrow \infty} \mathfrak{P}_{j}(y)$, using the Lebesgue's Dominated Convergence Theorem then

$$
\begin{array}{r}
\mathfrak{P}(\mathrm{y})-\frac{1}{|\mathrm{~B}|} \int_{\mathrm{B}} \mathfrak{P d} \mathrm{d} x=\lim _{j \rightarrow \infty} \frac{1}{\mathrm{~N} \omega_{N}} \int_{B} \mathfrak{H}_{j}\left(\mathfrak{P}_{j}(x)\right) \Theta(r, R) \mathrm{d} x, \\
r=|x-y| .
\end{array}
$$

This elementary result involves several strong indeterminations.

Lemma 4.7 (Lemma 3.1.1 page 113 [45]). Let $u \in W^{1, p}\left[\mathrm{~B}\left(\mathrm{x}_{0}, \mathrm{r}\right)\right], \mathrm{p} \geq 1$, where $\mathrm{x}_{0} \in \mathbb{R}^{\mathrm{N}}$ and $r>1$. Let $0<\delta<r$. Then

$$
\frac{\int_{B\left(x_{0}, r\right)} u(y) d y}{r^{N}}-\frac{\int_{B\left(x_{0}, \delta\right)} u(y) d y}{\delta^{N}}=\frac{\int_{B\left(x_{0}, r\right)}\left[\nabla u(y) \cdot\left(y-x_{0}\right)\right] d y}{N r^{N}} .
$$

The Lebesgue's Dominated Convergence Theorem implies

$$
\begin{aligned}
\frac{\int_{B\left(x_{0}, r\right)} \mathfrak{P}(y) d y}{r^{N}}-\frac{\int_{B\left(x_{0}, \delta\right)} \mathfrak{P}(y) d y}{\delta^{N}}= & \lim _{j \rightarrow \infty}\left\{\frac{\int_{B\left(x_{0}, r\right)}\left[\nabla \mathfrak{P}_{j}(y) \cdot\left(y-x_{0}\right)\right] d y}{N^{N}}\right. \\
& \left.-\frac{\int_{B\left(x_{0}, \delta\right)}\left[\nabla \mathfrak{P}_{j}(y) \cdot\left(y-x_{0}\right)\right] d y}{N^{N}}\right\} .
\end{aligned}
$$

\subsection{Newtonian potentials.}

The theory of Newtonian potentials for distributions with compact support are defined on $\Omega \subset$ $\mathbb{R}^{N}, N \geq 1$. We shall make use of the following results:

Proposition 4.8 (Proposition 5 page 281 [11]). Let $\Omega$ be a regular bounded open set and let $\mathrm{u} \in \mathrm{C}^{2}(\Omega) \cap \mathrm{C}_{\mathfrak{n}}^{1}(\bar{\Omega})$ with $\Delta \mathrm{u} \in \mathrm{L}^{1}(\Omega)$. Then

$$
\mathfrak{u}=\mathfrak{u}_{0}+\mathfrak{u}_{1}+\mathfrak{u}_{2} \text { on } \Omega \text {, }
$$


where $\mathfrak{u}_{0}, \mathfrak{u}_{1}, \mathfrak{u}_{2}$ are the Newtonian potentials of the distributions $\mathrm{f}_{0}, \mathbf{f}_{1}, \mathbf{f}_{2}$ on $\mathbb{R}^{\mathrm{N}}$ defined by

$$
\begin{array}{r}
\left\langle f_{0}, \zeta\right\rangle=\int_{\Omega} \zeta \Delta u d x \\
\left\langle f_{1}, \zeta\right\rangle=\int_{\partial \Omega} \zeta\left(-\frac{\partial u}{\partial n}\right) d \gamma \\
\left\langle f_{2}, \zeta\right\rangle=\int_{\partial \Omega} \frac{\partial \zeta}{\partial n} u d \gamma .
\end{array}
$$

We note that $\mathrm{f}_{0}, \mathrm{f}_{1}, \mathrm{f}_{2} \in \mathscr{E}^{\prime}$ :

$f_{0}$ is an integrable function on $\mathbb{R}^{N}$ with support contained in $\bar{\Omega}$,

$\mathrm{f}_{1}$ is a measure on $\mathbb{R}^{\mathrm{N}}$ with support contained in $\partial \Omega$,

$f_{2}$ is a distribution of order 1 on $\mathbb{R}^{N}$ with support contained in $\partial \Omega$.

We say that $\mathrm{u}_{1}$ is the simple layer (respectively double layer) potential defined by the function $-\frac{\partial \mathrm{u}}{\partial \mathrm{n}}$ (respectively $u$ ) continuous on $\partial \Omega$.

We apply this results to our sequence $\left\{\mathfrak{P}_{j}\right\}_{\mathfrak{j}=1}^{\infty}$. Using Proposition 4.8, we have

$$
\mathfrak{P}_{j}=\mathfrak{P}_{0, j}+\mathfrak{P}_{1, j}+\mathfrak{P}_{2, j} \text { on } \Omega \text {, }
$$

where $\mathfrak{P}_{0, j}, \mathfrak{P}_{1, j}, \mathfrak{P}_{2, j}$ are the Newtonian potentials of the distributions $\mathfrak{f}_{0, j}, \mathfrak{f}_{1, j}, \mathfrak{f}_{2, j}$. Therefore

$$
\begin{aligned}
\Delta \mathfrak{P}_{0, j} & =\mathfrak{f}_{0, j} \text { on } \mathbb{R}^{N}, \\
\Delta \mathfrak{P}_{1, j} & =\mathfrak{f}_{1, j} \text { on } \mathbb{R}^{N}, \\
\Delta \mathfrak{P}_{2, j} & =\mathfrak{f}_{2, j} \text { on } \mathbb{R}^{N} .
\end{aligned}
$$

We obtain

$$
\begin{array}{r}
\lim _{j \rightarrow \infty}\left\langle\mathfrak{f}_{0, j}, \zeta\right\rangle=-\infty, \\
\lim _{j \rightarrow \infty}\left\langle\mathfrak{f}_{1, j}, 1\right\rangle=\int_{\partial \Omega} 1\left(-\frac{\partial \mathfrak{P}_{j}}{\partial n}\right) d \gamma=\infty, \text { simple layer potentials, } \\
\lim _{j \rightarrow \infty}\left\langle\mathfrak{f}_{2, j}, \zeta\right\rangle=\int_{\partial \Omega} \frac{\partial \zeta}{\partial n}\left(\lim _{j \rightarrow \infty} \mathfrak{P}_{j}\right) \mathrm{d} \gamma, \text { double layer potentials. }
\end{array}
$$

Proposition 4.9 (Proposition 2 page 278 [11]). Let $\mathrm{f} \in \mathscr{E}^{\prime}$ and let $\mathrm{u}$ be the Newtonian potential of $\mathrm{f}$. Then for every multi-index $\alpha \in \mathbb{N}$

$$
\frac{\partial^{\alpha} u}{\partial x^{\alpha}}(x)=\langle f, 1\rangle+O\left(\frac{1}{|x|^{n+|\alpha|-1}}\right) \text { when }|x| \rightarrow \infty .
$$

In particular

$$
\begin{array}{r}
\text { if } N \geq 3, \quad \lim _{|x| \rightarrow \infty} u(x)=0, \\
\text { if } N \geq 2, \quad \lim _{|x| \rightarrow \infty} \nabla u(x)=0 .
\end{array}
$$

The last proposition is useful in the description of the sequence of Newtonian potentials $\left\{\mathfrak{f}_{1, j}\right\}_{j=1}^{\infty},\left\{\mathfrak{f}_{0, j}\right\}_{j=1}^{\infty},\left\{\mathfrak{f}_{1, j}\right\}_{j=1}^{\infty}$ and $\left\{\mathfrak{f}_{2, j}\right\}_{j=1}^{\infty}$. 


\subsection{The spherical average.}

For $u$ in 56 with $\{0\} \in \Omega \subset \mathbb{R}^{N}, N \geq 2$, let $\bar{u}$ be the spherical average of $u$, i.e.,

$$
\bar{u}(r)=\frac{1}{\omega_{N} r^{N-1}} \int_{|x|=r} u(x) d \gamma_{x} .
$$

With the change of variable $x \rightarrow y$, we have

$$
\bar{u}(r)=\frac{1}{\omega_{N}} \int_{|y|=1} u(r y) d \gamma_{y},
$$

and

$$
\frac{\mathrm{d} \bar{u}}{\mathrm{dr}}=\frac{1}{\omega_{N}} \int_{|y|=1} \nabla u(r y) \cdot y d \gamma_{y}
$$

Hence

$$
\frac{d \bar{u}}{d r}=\frac{1}{\omega_{N}} \int_{|y|=1} \frac{\partial u}{\partial r}(r y) d \gamma_{y}=\frac{1}{\omega_{N} r^{N-1}} \int_{|x|=r} \frac{\partial u}{\partial r}(x) d \gamma_{x}
$$

that is

$$
\frac{\mathrm{d} \bar{u}}{\mathrm{dr}}=\frac{1}{\omega_{N} r^{N-1}} \int_{B(0, r)} \Delta u(x) d x
$$

Therefore from Theorem 2.2 it follows that

$$
\lim _{j \rightarrow \infty} \frac{d \overline{\mathfrak{P}}_{j}}{d r}=-\infty
$$

\section{Statement and proof of the main results.}

Our main Theorem states the concentration of compactness. It can be regarded as a classical counterpart of Helly's Selection Theorem in the space of functions of bounded point variations (Theorem 2.35 page 59 [22]).

Theorem 5.1. Let $\Omega$ be a bounded domain in $\mathbb{R}^{N}, N \geq 3$ and any sequence of functions $\left\{\mathfrak{u}_{j}\right\}_{j=1}^{\infty}$ in $\mathrm{C}^{2}(\Omega) \cap \mathrm{C}^{0}(\bar{\Omega})$ satisfying

$$
\begin{array}{r}
-\Delta \mathfrak{P}_{j} \geq-\Delta \mathfrak{u}_{j} \text { in } \Omega, \\
\mathfrak{P}_{j} \geq \mathfrak{u}_{j} \text { on } \partial \Omega .
\end{array}
$$

Then there exist a constant $\mathrm{C}$ depending only on the sequence $\{\mathfrak{P}\}_{\mathfrak{j}=1}^{\infty}$, such that $\mathbf{u}_{\mathfrak{j}} \leq \mathrm{C}$ for all $j=1, \ldots, \infty$.

Proof. It is a simple consequence of Theorem 3.3 page 33 in [18]. 
Theorem 5.2 (Strong concentration of compactness for Newtonian potentials). Let $\Omega$ be a bounded smooth domain in $\mathbb{R}^{N}, N>2$. Then there exist a sequence $\left\{f_{1, j}\right\}_{j=1}^{\infty} \in \mathscr{E}^{\prime}$ the space of distributions on $\mathbb{R}^{N}$ with compact support such that:

(i) $\mathfrak{f}_{1, j}=\Delta \mathfrak{P}_{j}$ on $\Omega$ in the sense of the distributions.

(ii) The sequence of functions $\left\{\mathfrak{P}_{j}\right\}_{j=1}^{\infty} \in \mathrm{C}^{\infty}(\bar{\Omega})$ is non decreasing and bounded.

(iii) $\lim _{j \rightarrow \infty} \Delta \mathfrak{P}_{j}=-\infty$ uniformly on $\Omega$.

(iv) $\mathfrak{P}_{j}$ is the Newtonian potential of $\mathrm{f}_{1, j}$ on $\Omega$.

(v) The simple layer potential a $\mathfrak{f}_{1, j} \in \mathscr{E}^{\prime}$ associated to $\mathfrak{P}_{\mathrm{j}}$ satisfies

$\lim _{j \rightarrow \infty}\left\langle\mathfrak{f}_{1, j}, 1\right\rangle=\int_{\partial \Omega} 1\left(-\frac{\partial \mathfrak{P}_{j}}{\partial n}\right) \mathrm{d} \gamma=\infty$.

(vi) $\lim _{j \rightarrow \infty}\left\|\Delta \mathfrak{P}_{j}\right\|_{\mathrm{C}^{\alpha}\left(\overline{\mathrm{B}\left(\mathrm{x}_{0}, \mathrm{R}\right)}\right)}^{\prime}=\infty$ for all $\mathrm{B}\left(\mathrm{x}_{0}, \mathrm{R}\right) \subset \subset \Omega$.

(vii) Solid mean value property.

$$
\begin{array}{r}
\mathfrak{P}(\mathrm{y})-\frac{1}{|\mathrm{~B}|} \int_{\mathrm{B}} \mathfrak{P} \mathrm{d} x=\lim _{j \rightarrow \infty} \frac{(-1)}{\mathrm{N} \omega_{\mathrm{N}}} \int_{\mathrm{B}} \Delta \mathfrak{P}_{j}(x) \Theta(r, R) \mathrm{d} x, \\
r=|x-y| .
\end{array}
$$

where

$$
\Theta(r, R)=\frac{1}{N-2}\left(r^{2-N}-R^{2-N}\right)-\frac{1}{2 R^{N}}\left(R^{2}-r^{2}\right),
$$

for $\mathrm{N}>2$, where $\omega_{\mathrm{N}}$ is the volume of the unit ball in $\mathbb{R}^{\mathrm{N}}$.

(viii) For $\mathrm{N}>2$ we have

$$
\begin{aligned}
\frac{\int_{B\left(x_{0}, r\right)} \mathfrak{P}(y) d y}{r^{N}}-\frac{\int_{B\left(x_{0}, \delta\right)} \mathfrak{P}(y) d y}{\delta^{N}}= & \lim _{j \rightarrow \infty}\left\{\frac{\int_{B\left(x_{0}, r\right)}\left[\nabla \mathfrak{P}_{j}(y) \cdot\left(y-x_{0}\right)\right] d y}{N r^{N}}\right. \\
& \left.-\frac{\int_{B\left(x_{0}, \delta\right)}\left[\nabla \mathfrak{P}_{j}(y) \cdot\left(y-x_{0}\right)\right] d y}{N \delta^{N}}\right\} .
\end{aligned}
$$

(ix) The spherical average $\overline{\mathfrak{P}}_{\mathrm{j}}$ satisfy

$$
\lim _{j \rightarrow \infty} \frac{d \overline{\mathfrak{P}}_{j}}{d r}=-\infty
$$

Proof. This theorem is a collection of results stated in the A primer analysis section.

Theorem 5.3 (Weak concentration of compactness for Newtonian potentials). Let $\Omega$ be a bounded smooth domain in $\mathbb{R}^{N}, N \geq 1$. Then there exist a sequence $\left\{\mathbf{f}_{1, j}\right\}_{j=1}^{\infty} \in \mathscr{E}^{\prime}$ the space of distributions on $\mathbb{R}^{\mathrm{N}}$ with compact support such that:

(i) $\mathbf{f}_{1, j}=\Delta \mathfrak{F}_{j}$ on $\Omega$ in the sense of the distributions.

(ii) The sequence of functions $\left\{\mathfrak{F}_{j}\right\}_{j=1}^{\infty} \in \mathrm{C}^{\infty}(\bar{\Omega})$ is non decreasing and bounded.

(iii) $\lim _{\mathfrak{j} \rightarrow \infty} \Delta \mathfrak{F}_{\mathfrak{j}}=-\infty$ in measure on $\Omega$.

(iv) $\mathfrak{F}_{\mathfrak{j}}$ is the Newtonian potential of $\mathbf{f}_{1, j}$ on $\Omega$.

(v) $\lim _{j \rightarrow \infty}\|\mathfrak{F}\|_{C^{1, \alpha}\left(\overline{\mathrm{B}\left(\mathrm{x}_{0}, R\right)}\right)}=\infty$ for all $\mathrm{B}\left(\mathrm{x}_{0}, \mathrm{R}\right) \subset \subset \Omega$. 
(vi) One dimensional mean value property.

$$
\mathfrak{F}(x)-\frac{1}{b-a} \int_{a}^{b} \mathfrak{F}(t) d t=\lim _{j \rightarrow \infty}\left\{\frac{1}{b-a}\left[\int_{a}^{x}(t-a) \mathfrak{F}_{j}^{\prime}(t) d t-\int_{x}^{b}(b-t) \mathfrak{F}_{j}^{\prime}(t) d t\right]\right\} .
$$

(vii) Solid mean value property.

$$
\begin{array}{r}
\mathfrak{F}(y)-\frac{1}{|\mathrm{~B}|} \int_{\mathrm{B}} \mathfrak{F} \mathrm{d} x=\lim _{j \rightarrow \infty} \frac{(-1)}{\mathrm{N} \omega_{N}} \int_{B} \Delta \mathfrak{F}_{j}(x) \Theta(r, R) \mathrm{d} x, \\
r=|x-y| .
\end{array}
$$

where

$$
\Theta(r, R)=\frac{1}{N-2}\left(r^{2-N}-R^{2-N}\right)-\frac{1}{2 R^{N}}\left(R^{2}-r^{2}\right)
$$

for $\mathrm{N}>2$ and

$$
\Theta(r, R)=\log \left(\frac{R}{r}\right)-\frac{1}{2}\left(1-\frac{r^{2}}{R^{2}}\right)
$$

for $\mathrm{N}=2$, where $\omega_{\mathrm{N}}$ is the volume of the unit ball in $\mathbb{R}^{\mathrm{N}}$.

(viii) For $\mathrm{N}>2$

$$
\begin{aligned}
\frac{\int_{B\left(x_{0}, r\right)} \mathfrak{F}(y) d y}{r^{N}}-\frac{\int_{B\left(x_{0}, \delta\right)} \mathfrak{F}(y) d y}{\delta^{N}}= & \lim _{j \rightarrow \infty}\left\{\frac{\int_{B\left(x_{0}, r\right)}\left[\nabla \mathfrak{F}_{j}(y) \cdot\left(y-x_{0}\right)\right] d y}{N r^{N}}\right. \\
& \left.-\frac{\int_{B\left(x_{0}, \delta\right)}\left[\nabla \mathfrak{F}_{j}(y) \cdot\left(y-x_{0}\right)\right] d y}{N \delta^{N}}\right\} .
\end{aligned}
$$

Proof. This theorem is a collection of results stated in the A primer analysis section.

$$
\text { Received: July 2014. Accepted: April } 2015 .
$$

\section{References}

[1] C. C. Aranda and T. Godoy. Existence and multiplicity of positive solutions for a singular problem associated to the p-laplacian operator. Electronic Journal of Differential Equations, Vol. 2004(2004), No. 132, pp. 1-15.

[2] C. C. Aranda. Bounds and compactness for solutions of second-order elliptic equations. Electronic Journal of Differential Equations, Vol. 2012 (2012), No. 134, pp. 1-5.

[3] C. C. Aranda. On the Poisson's equation $-\Delta \mathfrak{u}=\infty$. CUBO A Mathematical Journal, Vol. 15 N. 01, (151-158), March 2013.

[4] M. S. Birman and M. Z. Solomjak. Spectral theory of self-adjoint operators in Hilbert space. D. Reidel Publishing Company, Dordrecht, Holland 1987. 
[5] R. J. Blakely. Potential theory in gravity and magnetic applications. Cambridge University Press 1996.

[6] H. Brezis. Analyse fonctionnelle. Théorie et applications. Dunod, Paris 1999.

[7] L. M. Burko and A. Ori. Internal Structure of Black Holes and Space Time Singularities: Annals of the Israel Physical Society Vol. 13. Israel Physical Society 1997.

[8] S. Chandrasekhar. The mathematical theory of black holes. Oxford University Press 1983.

[9] M. G. Crandall, P. H. Rabinowitz and L. Tartar. On a Dirichlet problem with singular nonlinearity. Comm. Partial Differential Differential Equations 2 (2) 1977, 193-222.

[10] B. Dacorogna. Introduction to the calculus of varitions. Imperial College Press 2004.

[11] R. Dautray and J. L. Lions. Mathematical analysis and numerical methods for science and technology. Vol. 1 Physical Origins and classical methods. Springer Verlag 1990.

[12] N. Denegaar, J. M. Miller, J. Kennea, N. Gehrels, M. T. Reynolds, and R. Wijnands. The $X$-ray flaring properties of Srg $A^{*}$ during six years of monitoring with Swift. To appear in Astrophysical Journal.

[13] B. Fauser, J. Tolksdorf and E. Zeidler editors. Quantum Gravity Mathematical Models and Experimental Bounds. Birkhauser Verlag 2006.

[14] G. B. Folland. Real analysis. John Wiley \& Sons Inc. 1999.

[15] V. P. Frolov and A. Zelnikov. Introduction to Black Hole Physics. Oxford University Press 2011.

[16] G, Ghisellini, R. D. Ceca, M Volonteri, G. Ghirlanda, F. Tavecchio, L. Foschini, G. Tagliaferri, F. Haardt, G. Pareschi and J. Grindlay, . Chasing the heaviest black holes in active galactic nuclei. Monthly Notices of the Royal Astronomical Society 405: 387, (2010).

[17] J. Giacomoni and K. Saoudi. Multiplicity of positive solutions for a singular and critical problem. Nonlinear Analysis 71 (2009) 4060-4077.

[18] D. Gilbarg and N. S. Trudinger. Elliptic Partial Differential Equations of Second Order. Classics in mathematics reprint of 1998 edition Springer.

[19] K. Falconer. Fractal Geometry Mathematical Foundations and Applications. Second Edition, Wiley 2003.

[20] P. M. Fitzpatrick. Principles of Celestial Equations. Academic Press 1970.

[21] N. S. Koshlyakov, M. M. Smirnov and E. B. Gliner. Differential Equations of Mathematical Physics. North-Holland Publishing Company 1964. 
[22] G. Leoni. A first course in Sobolev spaces. AMS 2009.

[23] O. Lpez-Cruz, C. Aorve, M. Birkinshaw, D.M. Worrall, H.J. Ibarra-Medel, W.A. Barkhouse, J.P. Torres-Papaqui, V. Motta (2014). The Central Galaxy of Abell 85-The Largest Galaxy Core Ever Observed. Preprint.

[24] V. Maz'ya. Sobolev Spaces With Applications to Elliptic Partial Differential Equations. 2nd. Edition Springer 2011.

[25] N. J. McConnell et al. Two ten billion solar mass black holes at the centres of giant elliptical galaxies Nature 480 (7376): 2158, 2011.

[26] M. McDonald et al. A Massive, Cooling Flow Induced Starburst in the Core of a Highly Luminous Galaxy Cluster, Nature, Aug 2012.

[27] F. Melia. The Galactic Supermassive Black Hole. Princeton University Press 2007.

[28] C. H. Nelson. Black Hole Mass, Velocity Dispersion, and the Radio Source in Active Galactic Nuclei. The Astrophysical Journal 544 (2): L91, 2000.

[29] J. Nečas. Direct Methods in The Theory of Elliptic Equations. Springer-Verlag 2012.

[30] B. O'Neill. The Geometry of Kerr Black Holes. A. K. Peters Massachusetts 1995.

[31] R. Penrose. The Road to Reality. Jonathan Cape London 2004.

[32] M. H. P. M van Putten. Gravitational Radiation Luminus Black Holes and Gamma-Ray Burst Supernovae. Cambridge University Press 2005.

[33] D. Raine and E. Thomas. Black Holes. Imperial College Press, second edition 2009.

[34] V. D. Radulescu. Singular Phenomena in Nonlinear Elliptic problems. In Handbook of differential equations, stationary partial differential equations. Volume 4 edited by M. Chipot Elsevier 2007.

[35] D. A. Riechers, F. Walter, C. L. Carilli, and G. F. Lewis. Imaging The Molecular Gas in a $z=$ 3.9 Quasar Host Galaxy at 0."3 Resolution: A Central, SubKiloparsec Scale Star Formation Reservoir in APM 08279+5255. The Astrophysical Journal 690 (1): 463485, (2009).

[36] F. Sauvigny. Partial differential equations 1. Springer 2006.

[37] G. Scharf. Finite Quantum Electrodynamics. Springer Verlag 1988.

[38] G. Scharf. Quantum Gauge Theories A True Ghost Story. John Wiley \& Sons, Inc 2001.

[39] D. Shiga. Biggest black hole in the cosmos discovered. NewScientist.com news service (10 January 2008). 
[40] I. S. Sokolnikoff. Tensor Analysis Theory and Applications. New York John Wiley \& Sons 1951.

[41] H. Stephani, D. Kramer, M. Maccallum, C. Hoenselaers and E. Herlt. Exact Solutions of Einstein's. Second Edition, Cambridge University Press 2003.

[42] M. Struwe. Variational Methods: Applications to Nonlinear Partial Differential Equations and Hamiltonian systems. Springer 2000.

[43] J. L. Walsh, A. J. Barth, L. C. Ho and M. Sarzi. The M87 Black Hole Mass from Gas dynamical Models of Space Telescope Imaging Spectrograph Observations. The Astrophysical Journal 770 (2): 86, (June 2013).

[44] X. Wu, F. Wang, X. Fan, W. Yi, W. Zuo, F Bian, L. Jiang, I. D. McGreer, R. Wang, J. Yang, Q. Yang, D. Thompson and Y. Beletsky (25 February 2015). An ultraluminous quasar with a twelve-billion-solar-mass black hole at redshift 6.30. Nature 518

[45] W. P. Ziemer. Weakly Differentiable Functions. Springer-Verlag 1989.

[46] K. Zubovas, S. Nayakshin and S. Markoff. Sgr A* flares: tidal disruption of asteroids and planets? to appear in Mon. Not. R. Astron. Soc.

[47] W. Zuo, X. B. Wu, X. Fan, R. Green, R. Wang and F. Bian. Black Hole Mass Estimates and Rapid Growth of Supermassive Black Holes in Luminous z 3.5 Quasars. Preprint (2014). 